\title{
Las TIC como herramientas cognitivas
}

\author{
Oscar Yecid Aparicio Gómez ${ }^{1}$
}

Recibido: 20-02-2018

Aceptado: 23-04-2018

Jonassen (1987) afirma que la mejor representación de la perspectiva constructivista puede ser la de quienes promueven las herramientas cognitivas. Sus palabras recogen el tránsito entre la teoría del pensamiento constructivista y la práctica educativa propuesta en la sociedad del conocimiento. Igualmente, los entornos de aprendizaje interactivos (Hannafin, 1992) y los micromundos (Rieber, 1992) son tipos de "educación con ordenador" que permiten la implantación de ambientes acordes al fundamento constructivista. Jonassen (1994) plantea la necesidad de poner en marcha entornos que apoyen la construcción colaborativa del conocimiento desestimulando la competición entre los estudiantes para obtener prebendas. Asimismo, debe permitir plantear múltiples perspectivas o interpretaciones de la realidad sobre supuestos reales y auténticos (Jonassen, 1991). Estamos por tanto ante la construcción colaborativa del conocimiento, trascendiendo su mera reproducción (Pérez, 2013).

Por tanto, es fundamental determinar el verdadero uso de la tecnología como mediación del aprendizaje y sobre este efecto, algunos autores como Jonnasen, Campbell y Davidson (1994) argumentan que el proceso de aprendizaje es holístico. Esta afirmación permite revisar desde este enfoque, el uso de la tecnología como herramienta de aprendizaje, más que como un medio instruccional.

La adquisición e integración del conocimiento es un proceso constructivo (Viejo, Cabezas \& Martínez, 2013), de manera que cuando se utilizan las herramientas cognitivas, los aprendices se apropian mucho más de la construcción del conocimiento que de la reproducción del mismo. Por tanto, el estudiante accede a una gran variedad de interpretaciones, incluyendo aquellas que proceden de la tecnología para tener su propia interpretación del mundo. Esto implica que la tecnología

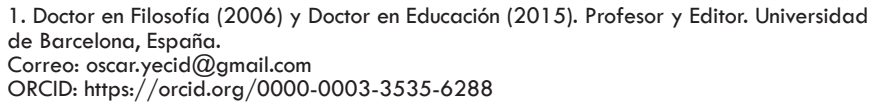


brinda un gran aporte a su propia representación del entorno real del estudiante (Ferreyra, 2014).

Las herramientas cognitivas son herramientas no inteligentes que recaen sobre el aprendiz para generar conocimiento, esto significa que hay una responsabilidad del estudiante, no del computador. Una de las mayores tecnologías aplicadas es el lenguaje, el cual amplifica el pensamiento del aprendiz. Los ordenadores pueden igualmente amplificar y reorganizar la forma en que aprenden los estudiantes (Pérez, 2014).

Las herramientas cognitivas deben ser pensadas como un conjunto de herramientas que los aprendices necesitan para sus procesos cognitivos en el aprendizaje, y que son el fundamento de la construcción de conocimiento con la tecnología, en la medida que un aprendizaje más significativo que si se reprodujera lo que un profesor transmite, empoderando así al estudiante para asumir su propio conocimiento (de Almeida, Santos \& Porto, 2016).

\section{Las herramientas cognitivas}

A través de historia, el ser humano ha desarrollado herramientas para facilitar y optimizar el trabajo físico y mental. Las herramientas han sido determinantes para la evolución humana (Engels, 1895) y para el desarrollo histórico y cultural de la humanidad. La herramienta, por tanto, ha transformado la manera de entender la actividad humana, el trabajo y la proyección de las labores cotidianas. La extensión de la inteligencia humana a través de instrumentos ha permitido considerar las herramientas cognitivas como amplificadoras de la mente (Rheingold, 1985) y reorganizadoras de la mente (Pea, 1985). Rheingold afirma que amplifican el pensamiento del estudiante al trascender las limitaciones cognitivas, y según Pea, además contribuyen a reorganizar los procesos cognitivos de las personas en la medida en que hacen uso de ellas.

Las herramientas cognitivas se refieren a aprender con la tecnología (Jonassen, 2000), evitando así caer en el enfoque de aprender de la tecnología, con el uso de programas de Enseñanza Asistida por Ordenador $(E A O)^{2}$. Quintana y Guioti (2006) ofrecen la siguiente clasificación de los 
usos de las TIC en los centros educativos: "Las TIC como contenidos u objetos de estudio, o sea, aprender sobre las TIC (...). Las TIC como "profesores", o sea, las TIC "enseñan", o sea, aprender de las TIC (...). Las TIC como herramientas para el aprendizaje, o sea, aprender con las TIC (...). Y "Las TIC como objeto de reflexión, o sea, aprender para las TIC". Salomon (1993) afirma que las herramientas son cognitivas siempre y cuando sirvan para ayudar a los estudiantes en la construcción de su propio pensamiento, superando las limitaciones cognitivas y proyectándolos a operaciones cognitivas superiores. Según Jonassen (2000) las herramientas cognitivas, o "mindtools" como las denomina en sus escritos, promueven la reflexión cognitiva como amplificadora, procurando así la construcción de las representaciones mentales de los estudiantes según el ámbito específico.

Jonassen (2000: 19) afirma que "el proceso de utilización de estas herramientas como formalismos de representación de las ideas las lleva a ser aprehendidas en las bases de los conocimientos personales para la integración de los computadores en las escuelas". Por tanto, las herramientas cognitivas representan una manera eficaz y eficiente para la integración de los computadores en la educación escolar, procurando involucrar a los estudiantes para que reflexionen sobre el contenido que están estudiando. El citado autor propone utilizar los recursos tecnológicos mediados por computadores como herramientas cognitivas porque potencian el pensamiento crítico, hacen más complejo el funcionamiento cognitivo y reestructuran el conocimiento que es generalizable a otras situaciones.

Aprender con la tecnología supone el uso y la implementación de las herramientas cognitivas en los procesos de aprendizaje suponiendo la disposición de un facilitador suficientemente preparado para que sea capaz de acompañar el camino del aprendiz y promueva en él la construcción del conocimiento, y no solo la reproducción de información. Por otra parte, urge la presencia de un aprendiz que desee aprender, que esté dispuesto a crear e innovar a partir de sus conocimientos previos y con el contacto con sus pares en su entorno vital.

Se requiere que el aprendizaje con la tecnología esté centrado en la persona del estudiante gracias al uso de las herramientas cognitivas que esta le brinda. La amplificación y reorganización permanente del conocimiento (Rheingold, 1985; y Pea, 1985) permitirá al aprendiz avanzar y profundizar en el conocimiento trascendiendo sus propias capacidades (Lajoie y Derry, 1993). 
Las tesis de los autores que hemos venido referenciando nos permiten afirmar que el uso de las herramientas cognitivas en la educación no solo privilegian la figura de la persona que aprende (Rodríguez Illera y Escofet, 2006), sino que el interés primordial debe ir en torno a los procesos de aprendizaje en vez de enfocarse casi exclusivamente en los métodos y procesos de enseñanza (Barrantes, Beltrán \& Pérez, 2106).

\subsection{Características de las herramientas cognitivas}

Fuertes (2011: 38-39) recoge algunas características de las herramientas cognitivas, citando a Jonassen (2000), y a Jonassen, Carr y Yueh (1998).

- Son herramientas de procesamiento cognitivo: Se trata de instrumentos centrados en el aprendiz, diseñados para promover el desarrollo de procesos mentales significativos, y no para sustituir la capacidad de reflexión de quien las usa. Por su parte, las herramientas cognitivas apoyan el pensamiento significativo cuando descargan la carga cognitiva del estudiante realizando las operaciones de bajo nivel cognitivo, permitiendo al usuario generar y comprobar hipótesis en situación de resolución de problemas.

- Son herramientas (no) inteligentes: No son sistemas destinados a decidir por el estudiante; no pretenden ser pensantes. Es decir, que no son programas que decidan cuánta instrucción ni de qué tipo debe ser suministrada al estudiante. La inteligencia la pone el estudiante, que es el encargado de crear, diseñar, planificar y construir su propio conocimiento.

- Son herramientas que promueven la colaboración cognitiva: Pueden funcionar como tecnología que favorezca los procesos mentales con el objetivo de ampliar el pensamiento del aprendiz y así poderlos reorganizar con facilidad. Pueden funcionar como compañero intelectual, disminuyendo y haciendo menos pesada la carga cognitiva del aprendiz, como por ejemplo los procesos memorísticos y representacionales. Así, el estudiante puede centrarse en el reconocimiento y la organización de la información. No se trata de que un programa presente información y vaya evaluando lo que el estudiante haya podido memorizar. 
Las características de las herramientas cognitivas privilegian en lugar del aprendiz en el centro de todo proceso educativo. La extensión de su pensamiento a través de artefactos que responden a sus intereses de aprendizaje facilita el propio proceso. Kennedy y McNaught (2001), refiriéndose a Duffy y Cunningham (1996) y Jonassen y Reeves (1996), amplían los rasgos de las herramientas cognitivas, concretando que son sencillas de utilizar por los estudiantes; permiten a los estudiantes dirigir preguntas significativas en un contexto realista y recibir un "feedback" apropiado, y a tiempo; alientan a los aprendices a ser responsables de su propio aprendizaje; descargan procesos cognitivos de bajo nivel para que el aprendiz pueda, mientras tanto, centrarse en procesos cognitivos de alto nivel; facilitan el desarrollo de estructuras más ricas y profundas de conocimiento; facilitan experiencias de aprendizaje colaborativo y de negociación de significados que permitan a los estudiantes explorar, comprobar, y validar sus concepciones.

\subsection{Tipologías de herramientas cognitivas}

Para Jonassen (2000) cada tipo de herramienta implica combinaciones distintas de pensamiento crítico, creativo y complejo. Por esta razón es importante presentar una tipología de las herramientas cognitivas que compartan unas características comunes, pero que a la vez ofrezcan una especificidad aplicable a sus usos prácticos en la educación. La clasificación de las herramientas cognitivas por computador está realizada en función del tipo de actividad que promueven y según se describe en Jonassen, Carr y Yueh (1998):

- Herramientas de interpretación de la información: Son herramientas de visualización que pueden ser interpretativas o expresivas. Ayudan a representar modelos mentales en imágenes. A través del uso de estas herramientas los estudiantes pueden sentirse estimulados para pasar de lo abstracto a lo real. Podemos señalar los laboratorios virtuales y los simuladores como ejemplos prácticos.

- Herramientas de modelado dinámico: Permiten que los estudiantes puedan describir relaciones dinámicas entre objetos, representen información cuantitativa, la calculen, manipulen, visualicen y reflexionen sobre ella, y finalmente organicen conjuntos de datos y los modifiquen tal como 
ocurre con las Hojas de cálculo como Excel de Microsoft, sistemas expertos (simuladores de resolución de problemas), micromundos (Rieber, 1992), entre otros.

- Herramientas de construcción de conocimiento: Integran diversos medios electrónicos, principalmente texto, sonidos e imágenes. Gracias a estas herramientas los estudiantes pueden experimentar como diseñadores, administrar proyectos, organizar y representar ideas propias, investigar y reflexionar sobre el trabajo realizado. En este grupo de herramientas podemos destacar las aplicaciones multimedia, editores de webs, sonido, imagen y video..., así como programas y aplicaciones específicas: YouTube, Picasa, Flickr, Scooch, Kartoo, etc.

- Herramientas de comunicación y colaboración: Su naturaleza socioconstructivista privilegia el aprendizaje colaborativo y la comunicación. A este tipo de herramientas corresponden ambientes sincrónicos y asincrónicos apoyados por computador y redes sociales. Requiere que los estudiantes puedan interpretar mensajes, razonen su contenido y puedan producir respuestas coherentes. En este grupo de herramientas se pueden encontrar los Chats, listas de correo, wikis, grupos de discusión, correo-e, etc., así como programas y aplicaciones específicas: Moodle, Meebo, Campfire, Facebook, Twiter, Instagram, Jotspot, Webbrush, Grouptivity, etc.

- Herramientas de organización semántica: Este sistema de registro estructurado de información sobre un tema puede usarse como herramientas para analizar y organizar un objeto de estudio. Gracias a esta herramienta los estudiantes pueden diseñar estructuras, clasificar la información y filtrar la información para transformarla en conocimiento. En este grupo de herramientas se pueden encontrar las bases de datos, esquemas, tablas y mapas conceptuales y mentales, así como programas y aplicaciones específicas como: CMapTools, Xmind, Bubbl.us, DebateMapper, FlowChart, XML/SWF Charts, Scribd, Google Drive, MySQL, SQL Server, etc.

Parece importante aclarar que esta clasificación (Jonassen, Carr y Yueh, 1998) no es exhaustiva con respecto a todas las herramientas que 
emergen a diario en la sociedad del conocimiento, por tanto, se hace imperioso mantener el estudio y la reflexión actualizados con respecto a las necesidades educativas de los estudiantes desde una óptica que contemple la innovación y la creatividad permanentemente.

Por otra parte, como ya hemos comentado, es a través la construcción activa del conocimiento, que este se hace más significativo, aplicable y recordable. Al utilizar herramientas cognitivas, el estudiante se involucra activamente en la interpretación del mundo externo y reflexiona sobre estas interpretaciones. El lugar que ocupan estos conocimientos, obtenidos desde la propia autoría, hace que permanezcan fijados en el tiempo; si fuese de otra manera, el estudiante vería obstaculizada su propia interpretación del suceso porque no podría compartir con el profesor un conjunto común de experiencias e interpretaciones previas.

En el caso de las TIC, estas se deben utilizar para que el estudiante aprenda mediante su acción diaria con la tecnología. Se hace necesario promover en el aula el desarrollo de tareas de recolección de datos de interés, manipulación de objetos digitales, presentación de hallazgos en distintos formatos, y especialmente a través del trabajo en equipo.

La tecnología brinda ahora un nuevo tratamiento para abordar la investigación escolar, siendo las Webquests una metodología que integra las TIC como TAC (Tecnologías para el Aprendizaje y el Conocimiento), propios en esta época de era digital, ya que permite organizar a los estudiantes en equipos de trabajo cooperativo, para plantear y resolver problemas, tareas o proyectos, que desde el uso de Internet les facilite la elaboración y compartición de productos de solución.

En este contexto pueden definirse la Webquest ${ }^{3}$, siguiendo las palabras de Quintana (2013), como un tipo de actividad didáctica basada en presupuestos constructivistas del aprendizaje y la enseñanza que se basa en técnicas de trabajo en grupo por proyectos y en la investigación como actividades básicas de enseñanza/aprendizaje. Las Webquest estimulan a los estudiantes no solo a adquirir información nueva, sino también a integrarla con la que ya poseen y a coordinarla con la conseguida por los compañeros para elaborar un producto o solucionar un problema. Para el campo de la investigación, debe contarse con nuevos conceptos

3. Podemos considerar las Webquest como una metodología para la enseñanza y el aprendizaje, basada en unidades didácTICas que integran las tecnologías digitales, que se desarrollan en entornos cooperativos. En las Webquests, "a partir de la presentación de una situación relativa a un tema y de su contexto, se propone al alumnado la realización en grupo de una tarea o de un proyecto, que conlleva la elaboración de un producto final con una presentación pública" (Quintana, 2013: 93). 
de organización del conocimiento en el entorno escolar que estimulen una mayor exploración y flexibilidad a partir de problemas concretos y relativos a sus propios intereses.

Desde otra perspectiva, se propone una tipología de usos de las TIC como instrumentos mediadores de las relaciones entre los estudiantes y el contenido; entre el profesorado y los contenidos; entre el profesorado y los estudiantes; en la actividad conjunta del profesorado y los estudiantes; y como instrumentos configuradores de entornos de trabajo y aprendizaje (Coll, Mauri, y Onrubia, 2008a). Esta perspectiva sugiere una humanización de las relaciones entre los actores educativos gracias a la mediación entre unos y otros y las TIC.

\subsection{Criterios pedagógicos}

Para fijar los objetivos del uso de las herramientas cognitivas en los procesos de aprendizaje acudimos a la mención que hace Fuertes (2011: 51) citando a Pea (1985: 20). Por tanto, los objetivos derivados del uso las herramientas cognitivas son: Establecer un nuevo énfasis en las habilidades cognitivas del uso de la información, incluyendo la definición de problemas, estrategias flexibles de recuperación y esquematización de información y hacer inferencias, resumir, e integrar diversos textos; poner el acento en la comunicación escrita y en las habilidades de búsqueda crítica, evaluar fuentes de información; Promover las habilidades metacognitivas y regulatorias como la planificación, el control de la comprensión, y el aprender a aprender; potenciar el desarrollo de estrategias para un pensamiento creativo y de resolución de problemas (brainstorming, descomposición de problemas, proponer, comprobar hipótesis), y métodos sistemáticos de toma de decisiones; enfatizar el trabajo cooperativo de resolución de problemas y las habilidades de negociación.

Las habilidades que emergen del uso de las herramientas cognitivas pueden consolidarse a partir de las buenas prácticas pedagógicas. La correcta ponderación en su aplicabilidad permitirá postular los criterios pedagógicos (Fuertes, 2011) que, según Jonassen (1996), deben seguir estas herramientas para alcanzar los objetivos ya mencionados:

- Capacidad de representación del conocimiento: la aplicación es utilizada como herramienta de representación del conocimiento. 
- Herramientas generalizables: La aplicación puede ser usada en algunas áreas de conocimiento.

- Promueven el pensamiento crítico: Implicación del aprendiz en el pensamiento crítico sobre un tema. Así el pensamiento es más profundo y de alto nivel.

- Aprendizaje transferible: Las destrezas que resultan del uso de las herramientas cognitivas son transferibles a otros campos de pensamiento.

- Pensamiento simple y formal: Las herramientas cognitivas deben promover el tipo de pensamiento requerido para construir las bases de conocimiento o para producir un material multimedia; es algo profundo.

- Las herramientas cognitivas son fáciles de aprender: El esfuerzo mental requerido para aprender a cómo utilizar el software no debería exceder los beneficios del resultado que nos permite obtener.

La capacidad de filtrar y reorganizar convenientemente la información a través de los usos correctos de las herramientas cognitivas permitirá generar conocimientos en los estudiantes, resultado de la construcción de su propio aprendizaje.

\section{El uso de las TIC en la educación escolar}

La escuela es una tecnología de la educación, y las tecnologías presentes en la cultura contribuyen en la definición de lo que en esa cultura se considera inteligencia (Lalueza, Crespo y Camps, 2008). Por tanto, todo lo que esté vinculado a la escuela, es decir, las aulas de clase, la pizarra, las libretas, ordenadores, tabletas, PDI, acceso a la web 2.0, EVEA (Entornos Virtuales de Enseñanza y Aprendizaje), etc., son creaciones tecnológicas que en su uso escolar tienen como función el cumplimiento de una tarea educativa, la mayoría de las veces para un gran número de personas simultáneamente (Sancho, 1998). Esta tecnología que determina lo que podemos considerar inteligencia se ha mantenido más o menos invariable durante siglos y a medida que aparecen herramientas innovadoras en 
otras áreas científicas, ha sido habitual que se incorporen a la tecnología educativa tradicional sin criterios demasiado claros.

La incorporación de las Tecnologías de la Información y la Comunicación a la Sociedad del Conocimiento ${ }^{4}$ ha generado una transformación en los escenarios educativos tradicionales, mientras aparecen otros nuevos, la mayoría de ellos con el interés del trabajo colaborativo. Coll y Rodríguez Illera (2008), afirman que la novedad y el interés de las TIC no residen en la introducción de un nuevo sistema de símbolos para representar la información, sino en el hecho de que permiten integrar los sistemas que ya conocemos creando entornos simbólicos que abren nuevas e insospechadas posibilidades para representar, procesar y difundir la información. Estas herramientas para la construcción del conocimiento permiten implicar a otros a través del aprendizaje colaborativo, motivando la innovación y la creatividad de los estudiantes y sus profesores.

Comprender el potencial pedagógico de las TIC e integrarlas en las estrategias de enseñanza se ha convertido en el gran reto de la educación actual. Su concepción artefactual y mediadora de las actividades educativas exige que sean consideradas en cuanto su orientación hacia fines donde su presencia adquiera significado. La función mediadora de las TIC se encuentra precisamente en que modifican el contexto tecnológico de la educación tradicional y lo enmarcan en una profunda transformación de los procesos de aprendizaje por su carácter menos rígido, más exploratorio, con una distribución del trabajo más flexible, una invitación permanente a la colaboración con otros, un medio idóneo para experimentar y reflexionar sobre la forma de aprender (Badía y Monereo, 2008).

La presencia de las TIC en la educación requiere el planteamiento de unos estándares para su optimización en los ámbitos de aprendizaje. Los National Educational Technology Standards del ISTE (International Society for Technology in Education), proponen seis estándares o referentes con sus respectivos indicadores ${ }^{5}$ :

4. El concepto de "sociedad del conocimiento" ha sido acuñado con el interés de resaltar que el conocimiento será cada vez más la base de los procesos sociales en todos los ámbitos de las sociedades. Por ejemplo, la importancia del conocimiento como recurso económico, que lleva irremediablemente a la necesidad de aprender a lo largo de toda la vida. A este respecto afirma Castells que "el conocimiento y la información son elementos decisivos en todos los modos de desarrollo, ya que el proceso de producción siempre se basa sobre cierto grado de conocimiento y en el procesamiento de la información. Sin embargo, lo que es específico del modo de desarrollo informacional es la acción del conocimiento sobre sí mismo como principal fuente de productividad". (Castells, 1996: 43)

5. Véase: http://www.iste.org/standards/iste-standards/standards-for-students 
- La creatividad y la innovación (con indicadores relacionados con el pensamiento creativo, la construcción del conocimiento y el desarrollo de procesos y productos innovadores mediante el uso de las TIC).

- La comunicación y la colaboración (con indicadores relacionados con la capacidad de trabajar y aprender colaborativamente utilizando herramientas TIC).

- La investigación y el manejo fluido de la información (con indicadores relacionados con el uso de las TIC para buscar, evaluar y utilizar información).

- El pensamiento crítico, la resolución de problemas y los procesos de toma de decisiones (con indicadores relacionados con la capacidad de pensar críticamente en la planificación y ejecución de planes y procesos de indagación utilizando herramientas y recursos $\mathrm{TIC}$ ).

- La ciudadanía digital (con indicadores relativos a la comprensión de temas relacionados con cuestiones cívicas, legales y éticas vinculados al uso de las TIC).

- Conceptos teóricos y funcionamiento de la tecnología (con indicadores relativos a la comprensión de conceptos, operaciones y sistemas tecnológicos).

Estos estándares con sus respectivos indicadores han de permitir plantear líneas de acción según el contexto educativo específico, mediante un proceso de diagnóstico y adaptación. Y dado que aún no se haya establecido un corpus teórico suficientemente sistematizado sobre el arribo de las TIC a la escuela (Area, 2005) el proceso de adaptación de la tecnología a los proyectos educativos de las instituciones escolares necesita una reflexión desde las políticas educativas de los países, la dirección de los centros, los estudiantes, los profesores y las familias. 


\section{Referencias bibliográficas}

Area, M. (2005). Las tecnologías de la información y comunicación en el sistema escolar. Una revisión de las líneas de investigación. Revista electrónica de Investigación y Evaluación Educativa, 11(1). Recuperado de http://www.uv.es/RELIEVE/v11n1/ RELIEVEV11n1_1.htm

Badía, A. y Monereo, C. (2008). La enseñanza y el aprendizaje de estrategias de aprendizaje en entornos virtuales. En Coll, C. y Monereo, C. (Eds.). Psicología de la educación virtual. Aprender y enseñar con las Tecnologías de la Información y la Comunicación (pp. 348 - 367). Madrid: Morata.

Barrantes, H. A., Beltrán, J. E. P., \& Pérez, F. A. R. (2016). Perfil del estudiante de pregrado de la Facultad de Estudios a Distancia de la Universidad Militar Nueva Granada. Revista Interamericana de Investigación, Educación y Pedagogía, RIIEP, 9(2). DOI: https://doi.org/10.22490/25391887.1948

Coll, C. y Rodríguez Illera, J. L. (2008). Alfabetización, nuevas alfabetizaciones y alfabetización digital: Las TIC en el curriculum escolar. En Coll, C. y Monereo, C. (Eds.). Psicología de la educación virtual. Aprender y enseñar con las Tecnologías de la Información y la Comunicación (pp. 325-347). Madrid: Morata.

Coll, C., Mauri, T. y Onrubia, J. (2008a). La utilización de las tecnologías de la información y la comunicación en la educación: Del diseño tecno-pedagógico a las prácticas de uso. En Coll, C. y Monereo, C. (Eds.). Psicología de la educación virtual. Aprender y enseñar con las Tecnologías de la Información y la Comunicación (pp. 74 103), Madrid: Morata.

Castells, M. (1996). La era de la información. Economía, Sociedad y Cultura. Madrid: Alianza Editorial.

de Almeida, R. R., Santos, M. F., \& Porto, J. C. (2016). Lectura de textos ficcionales y el enfoque escolar de literatura: contribuciones para una Pedagogía de la Elección. Revista Interamericana de Educación, Pedagogía y Estudios Culturales, 9(1), 35-51. DOI: https://doi.org/10.22490/25391887.1925

Duffy, T. y Cunningham, D. (1996). Constructivism: Implications for the design and delivery of instruction. En Jonassen, D. (Ed.). Handbook of Reasearch for Educational Communications and Technology (pp. 170-198) Nueva York: Simon and Schuster.

Engels, F. (1895-1896). El papel del trabajo en la transformación del mono en hombre. Die Neue Zeit, 2 (44). Recuperado de: https://www.marxists.org/ espanol/me/1870s/1876trab.htm

Ferreyra, H. A. (2014). Mesas Socioeducativas para la Inclusión y la Igualdad. Un programa "De todos con todos". Una experiencia en construcción. Revista Interamericana de Investigación, Educación y Pedagogía, RIIEP, 7(2). DOI: https://doi. org/10.15332/s1657-107X.2014.0002.01 
Fuertes, M. (2011). El disseny i la creació de llibres electrònics educatius: usos de Lektor com una eina cognitiva. (Tesis doctoral no publicada). Barcelona: Universitat de Barcelona.

Hannafin, M. J. (1992). Emerging technologies, ISD, and learning environments: Critical perspectives. Educational Technology Research and Development, 40(1), 49-63.

Jonassen, D. H. (1987). Mindtools in schools. Nueva York: Macmillan Publishing.

Jonassen, D. H. (1991). Evaluating constructivistic learning. Educational Technology, 31(9), 28-33.

Jonassen, D. H. (1994). Thinking Technology: Toward a constructivist design model. Educational Technology, 34(4), 34-37.

Jonassen, D. H. (1996). Computers in the Classroom: Mindtools for Critical Thinking. Englewood Cliffs, NJ: Prentice Hall.

Jonassen, D. H. (2000). Computers as Mindtools for Schools: Engaging Critical Thinking. New Jersey: Prentice - Hall.

Jonassen, D., Campbell, J., y Davidson, M. (1994). Learning with media: Restructuring the debate. Educational Technology Research and Development 42(2). 31-39.

Jonassen, D. y Reeves, T. C. (1996). Learning with technology: Using computers as cognitive tools. En Jonassen, D. (Ed.). Handbook of research for educational communications and technology (pp. 693-719). New York: Macmillan.

Jonassen, D., Carr, C. y Yueh, H. (1998). Computers as Mindtools for engaging learners in critical thinking. TechTrends, 43(2), 24-32.

Kennedy, D. y McNaught, C. (2001). Computer-based cognitive tools: Description and design. En Montgomerie, C. y Viteli, J. (Eds). ED-MEDIA 2001. Proceedings of the 13th Annual World Conference on Educational Multimedia, Hypermedia y Telecommunications. Tampere, Finland, 25-30 June (pp. 925-930). Norfolk, VA: Association for the Advancement of Computers in Education (AACE).

Lajoie S. y Derry, S. (1993). Computers as cognitive tools. New Jersey: Lawrence Erlbaum Associates.

Lalueza, J. L., Crespo, I. y Camps, S. (2008). Las tecnologías de la información y la comunicación y los procesos de desarrollo y socialización. En Coll, C. y Monereo, C. (eds.). Psicología de la educación virtual (pp. 54 - 73). Madrid: Morata, 2008.

Pea, R. (1985). Beyond amplification: Using the computer to reorganize mental functioning. Educational Psychologist, 20(4), 167-182. 
Pérez, T. H. P. (2013). Aproximaciones al estado de la cuestión de la investigación en educación y derechos humanos. Revista Interamericana de Investigación, Educación y Pedagogía, RIIEP, 6(1). DOI: https://doi.org/10.15332/s1657-107X.2013.0001.05

Pérez, T. H. (2014). Colombia: de la educación en emergencia hacia una educación para el posconflicto y la paz. Revista Interamericana de Investigación, Educación y Pedagogía, RIIEP, 7(2). DOI: https://doi.org/10.15332/s1657-107X.2014.0002.06

Quintana, J. (2013). La curación o responsabilidad de contenidos, el profesorado como organizador del proceso de aprendizaje mediante Webquests. En Bergmann, J. y Grané, M. (2013). La universidad en la nube. A universidade na nuvem (p. 89-105). Barcelona: LMI, Universitat de Barcelona,. Recuperado de http://www.Imi.ub.edu/ transmedia21/vol6/

Quintana, J. y Guioti, E. (2006). Algunes reflexions sobre las TIC a l'educació des de la perspectiva de Paulo Freire i una actuació concreta. Tarragona: URV/Edutec.

Rheingold, H. (1985). Tools for thought. MIT Press. Recuperado de http://www. rheingold.com/texts/tft/

Rieber, L. (1992). Computer - based microworlds: A bridge between constructivism and direct instruction. Educational Technology. Research and Development, 40(1), 93-106.

Rodríguez Illera, J. L. y Escofet, A. (2006). Aproximación centrada en el estudiante como productor de contenidos digitales en cursos híbridos. Revista de Universidad y Sociedad del Conocimiento (RUSC), 3 (2). Recuperado de http://www.uoc.edu/rusc/3/2/dt/esp/ rodriguez_escofet.pdf

Salomon, G. (1993). On the nature of pedagogic computer tools. The case of the writing partner. En Lajoie, S. P. y Derry, S. J. (Eds.). Computers as cognitive tools (pp. 179-198). Hillsdale, NJ: Lawrence Erlbaum Associates.

Sancho, J. M. (1998). Para una Tecnología Educativa. Barcelona: Horsori.

Viejo, C. M., Cabezas, I. L., \& Martínez, M. D. J. I. (2013). Las redes de académicas en la docencia universitaria. Revista Interamericana de Investigación, Educación y Pedagogía, RIIEP, 6(2). DOI: https://doi.org/10.15332/s1657-107X.2013.0002.03 